\title{
Áreas del conocimiento de apoyo para la gestión de proyectos de profesores universitarios venezolanos
}

\author{
Support knowledge areas for project management of venezuelan \\ university professors
}

RECIBIDO 1/4/2019 ACEPTADO 4/8/2020 PUBLICADO 1/12/2020

\section{María Daniela Gómez Suárez}

Universidad Simón Bolívar - Sede Litoral, Venezuela

mdgomez@usb.ve

\section{RESUMEN}

Todo profesor universitario se enfrenta constantemente a proyectos. Estos profesionales deben considerar que el impacto de cualquier actividad o proyecto que realizan supera el ámbito personal o de la institución donde trabajan, influyendo en la sociedad como un todo, debiendo optimizar la gestión de los mismos. Dentro de la metodología propuesta por el Project Management Institute, se consideran las denominadas áreas de conocimiento que son un conjunto de nociones de diversas áreas, de las cuales se toman técnicas y herramientas, útiles para gestionar proyectos. Las áreas de conocimiento se clasifican en tres tipos: medulares, de integración y de apoyo, siendo estas últimas el centro del presente artículo, el cual tiene como objetivo general diagnosticar la gestión realizada en áreas de conocimiento de apoyo de los profesores del Departamento de Tecnología de Servicios de la Universidad Simón Bolívar de Venezuela. Para este diagnóstico, se utilizó un cuestionario de autoevaluación donde se identificaron potenciales mejoras y necesidades de formación particulares en las áreas de conocimiento de apoyo relacionadas con la gestión de los recursos humanos, de las comunicaciones, de los riesgos, de las adquisiciones y de los interesados.

PALABRAS CLAVE gestión, proyectos, áreas de conocimiento, apoyo.

\section{ABSTRACT}

Every university professor constantly faces projects. These professionals must consider that the impact of any activity or project they carry out exceeds the personal scope or the institution where they work, influencing society as a whole, it is so they must optimize their management. Within the methodology proposed by the Project Management Institute, are considered the so-called areas of knowledge which are a set of notions of various areas, from which techniques and tools are taken, useful for managing projects. The areas of knowledge are classified into three types: essential, integration and support, being these lasted the center of the present article, which has as general objective to diagnose the management in areas of knowledge of support of the professors of the Service Technology Department of the Simón Bolívar University from Venezuela. For this diagnosis, a self-assessment questionnaire was used where potential improvements and training needs were identified to the areas of knowledge of support related to the management of human resources, communications, risks, acquisitions and stakeholders.

KEYWORDS management, project, knowledge areas, support. 


\section{INTRODUCCIÓN}

La gestión de proyectos presenta una serie de retos que requieren que los encargados de los mismos, así como su equipo de trabajo, utilicen metodologías adecuadas para tal fin. Si el ámbito de realización de los proyectos son las universidades, donde el impacto o resultados de los mismos son extensibles hacia toda la sociedad, con mayor razón la gestión debe apoyarse en las herramientas y técnicas apropiadas. Esto no ocurre siempre, ya que la formación académica de los miembros de la comunidad universitaria usualmente no se vincula con temas gerenciales y de gestión de proyectos, a menos que sus carreras de pregrado y de postgrado lo estipulen o se centren en ello.

A pesar de lo antes señalado, es posible que, de forma intuitiva, algunos profesores estén aplicando metodologías, técnicas y herramientas útiles para gestionarlos, específicamente las vinculadas con las áreas de conocimiento de la gestión de proyectos que se aproximen a su formación académica. El presente estudio se centra en los profesores del Departamento de Tecnología de Servicios de la Universidad Simón Bolívar de Venezuela que realizan proyectos de investigación (dado su tipo de contratación, solo profesores ordinarios o contratados a dedicación exclusiva o tiempo integral) para determinar las fallas en preparación para la gestión de proyectos y así poder concretar las necesidades de formación en el área. Por consiguiente, el objetivo general de este artículo es diagnosticar la gestión realizada en áreas de conocimiento de apoyo en proyectos realizados por los profesores del departamento antes referido.

Antes de explicar en qué consisten las áreas de conocimiento de apoyo de la gerencia de proyectos, es necesario iniciar definiendo que un proyecto. Definimos el mismo como "la búsqueda de una solución inteligente al planteamiento de un problema, la cual tiende a resolver una necesidad humana" (Baca, 2013, p. 2), lo que implica que además de poseer competencias en áreas específicas de cada proyecto que se desea desarrollar, también se necesitan competencias particulares en la gestión de procesos y de las áreas de conocimiento de los proyectos.

Basado en lo planteado por Wallace (2014) y el Project Management Institute (2013), las principales características de los proyectos se pueden resumir como sigue:

- Son únicos, ya que tienen un propósito u objetivo específico.

- Se caracterizan por el cambio, ya que se llevan a cabo para obtener un resultado tangible o intangible de características únicas.

- Tienen una serie de restricciones operativas, así como objetivos de desempeño individuales.

- Disponen de recursos limitados, entre ellos el tiempo, lo que hace que posean una duración limitada que los hace finitos. Dicha temporalidad no se aplica al producto, servicio o resultado creado por el proyecto, si no a las actividades llevadas a cabo para alcanzarlos.

- Los equipos de proyectos son únicos y multidisciplinares.

- A pesar de la planificación, sus resultados pueden ser desconocidos, dado el grado de incertidumbre que los rodean debido a la interacción con distintas variables organizacionales y del entorno.

- Su ciclo de vida implica el tránsito por fases de desarrollo concisas vinculadas a actividades, objetivos específicos y procesos de gestión.

- Se llevan a cabo siguiendo una secuencia de actividades jerárquicas e interdependientes entre sí, que permiten alcanzar hitos en cada fase del proyecto. 
- Tienen un alto nivel de riesgo, dada la incertidumbre en la que se realizan y las restricciones de recursos que limitan las pruebas de ensayo y error para conocer previamente los resultados. En estas circunstancias, los errores pueden ser muy costosos para los proyectos.

- Son diversos los mercados ante los cuales pueden ofrecer respuestas, por lo que pueden ser de consumo particular de una organización o persona, así como de consumo masivo. Dado lo antes señalado, pueden tener implicaciones privadas o sociales.

- Pueden ser secundarios a la función principal de una organización, pero siempre vinculados a su planificación estratégica, para así mejorar la eficiencia organizacional.

Dadas las características antes mencionadas, gestionarlos supone realizar los procesos que implican definir, planificar, organizar, controlar y liderar un proyecto hasta que el resultado final sea alcanzado, evidenciando que son más complejos que las operaciones rutinarias que puede realizar cualquier organización, lo que requiere la aplicación de técnicas y herramientas que permiten llevar a término, y según lo planeado, cualquier proyecto emprendido (Gómez, 2011). Para lograr esto, se aplican los cinco grupos de procesos (Project Management Institute, 2013) que interactúan entre sí mientras dure el proyecto, los cuales se relacionan con:

- Iniciación: engloba todas aquellas actividades o tareas que hay que realizar para convertir una idea en un proyecto viable (Ollé y Cerezuela, 2017). Para ello se define y/o autoriza el proyecto o una nueva fase del mismo. Este proceso es muy importante ya que, como señala Turner (2016), si un proyecto no se inicia bien es poco probable que termine bien. Por lo tanto, iniciar un proyecto apropiadamente es algo en lo que se debe poner bastante esfuerzo.

- Planificación: establecimiento del curso de acción requerido para alcanzar el resultado, definiendo el alcance y refinando los objetivos. Para Marchewka (2015, p. 91) este proceso requiere una "mentalidad más estratégica y táctica" para identificar, coordinar y autorizar las actividades que deben realizarse, es decir, que deben "diseñarse apropiadamente y desarrollarse todas las herramientas de gestión de proyectos requeridas" (Wilson, 2015, p. 14).

- Ejecución: es la integración de recursos para llevar a cabo el plan de gestión del proyecto a fin de satisfacer las especificaciones del mismo, completando el entregable del proyecto (Wilson, 2015). Esta integración requiere de un esfuerzo para lograr una sinergia con los diferentes actores y poder cumplir con el trabajo establecido o planificado (Prieto, 2015).

- Monitoreo y control: supervisión y regulación del avance del proyecto a fin de identificar variaciones respecto al plan de gestión e iniciar los cambios o "cualquier acción correctiva en caso de ser necesario" (Wallace, 2014, p. 22), con la finalidad de que el proyecto no se desvíe de sus objetivos iniciales (Estrada, 2015).

- Cierre: además de la finalización de todas las actividades del proyecto o de una fase del mismo, este grupo de procesos da formalidad a la aceptación del producto final, de forma que el proyecto o la fase pueda cerrarse de manera ordenada (Marchewka, 2015) y, por lo tanto, traspasar a los clientes o a la siguiente fase los entregables. Como afirman Ollé y Cerezuela (2017), además del cierre administrativo (consiste en comprobar la realización de todos los pagos, la conciliación de cuentas y la transición del equipo de trabajo a otras tareas o proyectos), debe documentarse la información administrativa y técnica del proyecto, es decir, recoger las lecciones aprendidas. 
Por su parte, las áreas de conocimiento en la gerencia de proyectos representan "un conjunto completo de conceptos, términos y actividades que conforman un ámbito profesional" (Project Management Institute, 2013, p. 60) y que comprenden las herramientas y técnicas necesarias para obtener resultados en cada grupo de procesos, además de "definir las variables que intervienen en un proyecto y que inciden en el producto, servicio o resultado esperado del proyecto" (Ugas, 2008, p. 78). Las áreas de conocimiento son de tres tipos: integradoras (gestión de la integración de todas las áreas), medulares (gestión del alcance, gestión del tiempo, gestión de los costos y gestión de la calidad), y facilitadoras o de apoyo (gestión de los recursos humanos, de las comunicaciones, de los riesgos, de las adquisiciones y de los interesados).

A continuación, se resumen las áreas de conocimiento de apoyo o facilitadoras para la gestión de los proyectos (en las cuales se enfoca el presente trabajo):

- Gestión de los recursos humanos: se realiza para organizar, integrar y dirigir al equipo del proyecto, lo que tiene una importancia significativa ya que el capital humano es la fuente primaria de trabajo (Estrada, 2015). Como aportan Romano y Yacuzzi (2011), la administración de los recursos humanos en los proyectos y su gestión implica la asignación de roles y responsabilidades que permitan concretar los fines, así como involucrar a dicho recurso en la planificación y toma de decisiones del proyecto, lo que incrementará su compromiso, experiencia y participación.

- Gestión de las comunicaciones: se realiza para garantizar que toda la información requerida por todos los involucrados en un proyecto sea recopilada, almacenada y distribuida de forma oportuna y por los medios adecuados, para que haya confort y confianza durante todo el proyecto (Turner, 2016). Este es un aspecto importante a considerar, ya que como afirman Sebastián et al. (2017), tan importante como tomar las decisiones adecuadas es que éstas lleguen a las personas adecuadas.

- Gestión de los riesgos: se realiza para identificar, analizar, planificar, anticiparse y controlar las respuestas ante posibles situaciones de riesgo, todo ello basado en "ideas conceptuales de los entregables del proyecto" (Wilson, 2015, p. 45). El riesgo siempre debe ser controlado para evitar al máximo eventos que, programados o no, signifiquen un problema para el proyecto (Estrada, 2015).

- Gestión de las adquisiciones: consiste en una serie de operaciones conjuntas y vinculadas que permitan incorporar los recursos materiales que permitan desarrollar las actividades de un proyecto (Sebastián et al., 2017). Además, esta gestión incluye la renta o contratación de todos aquellos bienes y servicios requeridos por el equipo de trabajo y que sean "necesarios para que el proyecto evolucione" (Estrada, 2015, p. 86).

- Gestión de los interesados: considerando lo planteado por el Project Management Institute (2013, p. 391), con esta gestión se identifican a las "personas, grupos u organizaciones que pueden afectar o ser afectados por el proyecto" para así analizar sus expectativas y desarrollar estrategias basadas en su posible impacto en el proyecto. Sin embargo, como señalan Holloway et al. (2015), este es un proceso que debe ir más allá y con el cual se debe tratar con los involucrados lo más que se pueda.

Tal como plantean Mariné y Rodriguez (2014), citados por Ollé y Cerezuela (2017) y el Project Management Institute (2013), la vinculación entre procesos de gestión de proyectos y áreas de conocimiento de apoyo puede observarse en la figura 1 , siendo esa relación la que permite realizar el diagnóstico del presente estudio. 
FIGURA 1. Vínculo entre grupos de procesos y áreas del conocimiento de apoyo

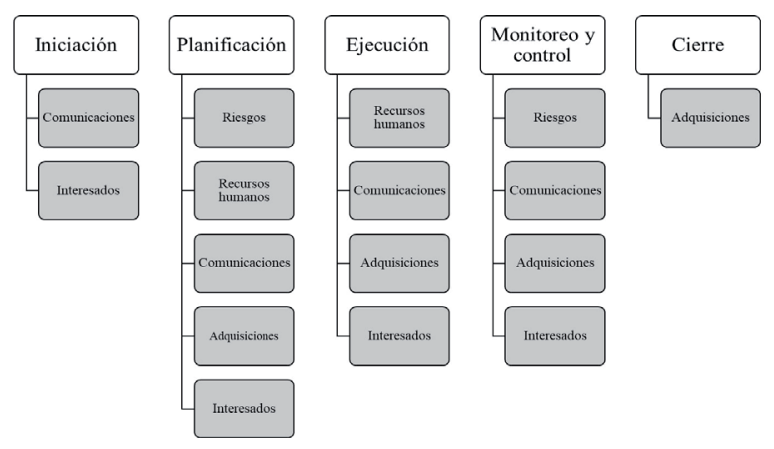

Aunque no forma parte del presente artículo, el diagnóstico realizado a los profesores sirve como punto de partida para diseñar un plan de formación en gestión de proyectos para los docentes de la universidad, porque tal como señalan Santos et al. (2013, p. 66), cada vez existe una "mayor preocupación por la actualización y ampliación de los conocimientos adquiridos por el profesorado durante su formación inicial, así como por la adquisición de aquellas competencias o destrezas que puedan ser precisas a lo largo de su carrera profesional" y, en el caso de la gestión de proyectos, se hace necesario formar a los profesores para mejorar su desempeño laboral.

\section{MÉTODO}

La presente investigación, de tipo descriptivo con un diseño no experimental, se realiza para diagnosticar la gestión en áreas de conocimiento de apoyo en proyectos realizados por los profesores del Departamento de Tecnología de Servicios de la Universidad Simón Bolívar para así "establecer su estructura o comportamiento" (Arias, 2012, p. 24) ante los proyectos que han llevado a cabo dentro de la institución educativa.

La población en estudio son los profesores del referido departamento universitario que, por su tipo de contratación, realizan proyectos en la universidad (sean estos a tiempo integral o a dedicación exclusiva). Se realizó un muestreo intencional para seleccionarlos a ellos, dejando de lado a aquellos profesores convencionales o contratados por servicios profesionales cuya dedicación solo requiere su presencia en la institución para impartir clases por horas. Se consideró el muestreo intencional ya que con este se seleccionan "casos, de personas o de situaciones, a partir de criterios teóricos y no estadístico, que den información importante para responder las preguntas de investigación” (Fàbregues et al., 2016, p. 207).

El instrumento de recolección de datos es un cuestionario de autoevaluación propuesto por Palacios (2009), en el cual, para cada área de conocimiento y cada proceso de la gestión de proyectos, se propone una serie de preguntas, las cuales se evalúan considerando una ponderación en escalas que son:

$$
\begin{aligned}
& 1=\text { Deficiente } \\
& 2=\text { Regular } \\
& 3=\text { Básico } \\
& 4=\text { Bien } \\
& 5=\text { Excelente } \\
& \text { NA = No aplica }
\end{aligned}
$$

Para la fecha de realización del cuestionario, el Project Management Institute aún no consideraba como décima área de conocimiento la gestión de los interesados, pero de igual modo se consideraron preguntas que cubrieran esa área, por lo que se consideró apto para diagnosticar la gestión de los profesores en todas las áreas de conocimiento (el caso de las áreas integradora y medulares será presentado en otro trabajo). 
La autoevaluación se realiza según la experiencia gestionando proyectos y mediante sus respuestas, considerando las escalas antes mencionadas, a las siguientes cuestiones por área de conocimiento:

- Gestión de los recursos humanos:

- Planificación de la organización: se detectaron roles requeridos para cumplir adecuadamente con las distintas tareas identificadas.

- Reclutamiento del personal: se buscaron y asignaron responsables con el perfil adecuado para las distintas tareas.

- Desarrollo del equipo: se trabajó en mejorar la efectividad del equipo por medio de entrenamiento, la distribución física, la motivación y otras acciones que contribuyeran al buen trabajo.

- Evaluación del desempeño: se realizó algún tipo de evaluación del desempeño de los participantes del proyecto, favoreciendo su mejora profesional.

- Gestión de las comunicaciones:

- Planificación de las comunicaciones: se identificaron las necesidades de información de los involucrados.

- Distribución de la información: los miembros del equipo estaban debidamente informados, sabiendo dónde o cómo conseguir los datos que requerían.

- Reportes de progreso: se realizaron reportes periódicos y reuniones para mantener informados a los distintos actores.

- Cierre administrativo: se realizó un cierre final que permitiera recoger, mediante un sistema para manejar la información, los principales aprendizajes del proyecto.

- Gestión de los riesgos:

- Identificación de riesgos: se determinaron que sucesos de riesgo pueden afectar a la efectividad del proyecto, usando listas de chequeo, tormentas de ideas, etc.

- Calificación: se evaluó la probabilidad de que ocurrieran los eventos riesgosos detectados y su impacto o efecto.

- Plan de respuesta: se diseñaron planes de respuesta para adelantarse a los riesgos, ya sea para mitigar o absorber el riesgo.

- Control de respuestas: se hicieron revisiones periódicas de riesgos, verificando posibles riesgos y activando contingencias.

- Gestión de las adquisiciones:

- Plan de compras: se creó un plan de compras que identificara los materiales o subcontratos que requieren para hacer sus proyectos.

- Plan de requerimientos: se diseñó la manera para realizar las compras en función de las necesidades detectadas.

- Ciclo de solicitación: se hizo un buen proceso, buscando proveedores, obteniendo ofertas y eligiendo al más adecuado.

- Administración de contratos: se efectuó una labor eficiente en el manejo e inspección de los contratos, con algún sistema para hacerle seguimiento. 
- Cierre de contratos: se realizó adecuadamente el cierre de los contratos otorgados y la rendición de cuentas de los mismos.

- Gestión de los interesados:

- Planificación del alcance: se empleó una metodología para definir el alcance del proyecto, considerando distintos actores, usuarios, clientes e interesados en los resultados.

- Estimación de duración: se empleó algún criterio para asignar tiempos de ejecución en consulta con los involucrados.

- Programación de actividades: se construyó un cronograma coherente que permitiera ver cuando se harían las actividades.

- Estimación de los costos: se prepararon estimaciones de costos empleando información y métodos de estimación consistentes.

- Planificación de la calidad: se especificaron los resultados que deben ofrecer los productos finales con indicadores de gestión.

- Plan integral: se preparó un plan integral y coherente que considerara las distintas áreas de la gerencia de proyectos (alcance, tiempo, costos, calidad, recursos humanos, comunicaciones, riesgos, compras e involucrados).

\section{RESULTADOS}

A continuación, se presentan los resultados obtenidos tras la autoevaluación realizada por los profesores del Departamento de Tecnología de Servicios de la Universidad Simón Bolívar, para cada área de conocimiento de apoyo con sus procesos vinculados.

Como puede apreciarse en la figura 2, relacionada con la gestión de los recursos humanos del proyecto, las respuestas de los profesores consultados indican que la mayoría en sus proyectos no contaron con un equipo de trabajo a la hora de realizarlos. Por lo tanto, el grueso de las respuestas obtenidas para cada ítem indica que no aplica para sus casos particulares. Esto puede deberse en gran medida a que la universidad impulsa la realización de proyectos de forma individual, especialmente para aquellos profesores noveles, así como para quienes aún no ingresan al escalafón universitario, siendo este el caso mayoritario de quienes hacen vida en el departamento estudiado.

A la hora de planificar el equipo, a través de los roles necesarios para cada miembro y proyecto, destaca que prácticamente un tercio de los consultados hicieron una gestión autoevaluada como regular y básica, por lo que no dejaron claras las expectativas y papel que debieron cumplir quienes participaron en sus equipos durante la realización de sus proyectos.

Si se trata de la asignación de las personas más idóneas para la realización de las tareas requeridas, el grueso de las respuestas se centró en indicar una gestión deficiente o, por el contrario, buena, lo que concatenando con la respuesta anterior indica que nunca contaron a la vez con los roles y responsabilidades debidamente definidos e integrados para el equipo de trabajo, lo que probablemente tuvo un impacto negativo en la ejecución del proyecto.

De forma regular y bien fue mayoritariamente catalogado el desarrollo del equipo de trabajo, siendo este un resultado no esperado dadas las carreras dictadas por los profesores del departamento (entre ellas 
Figura 2. Gestión de los recursos humanos en proyectos realizados por profesores del Departamento de Tecnología de Servicios - USB

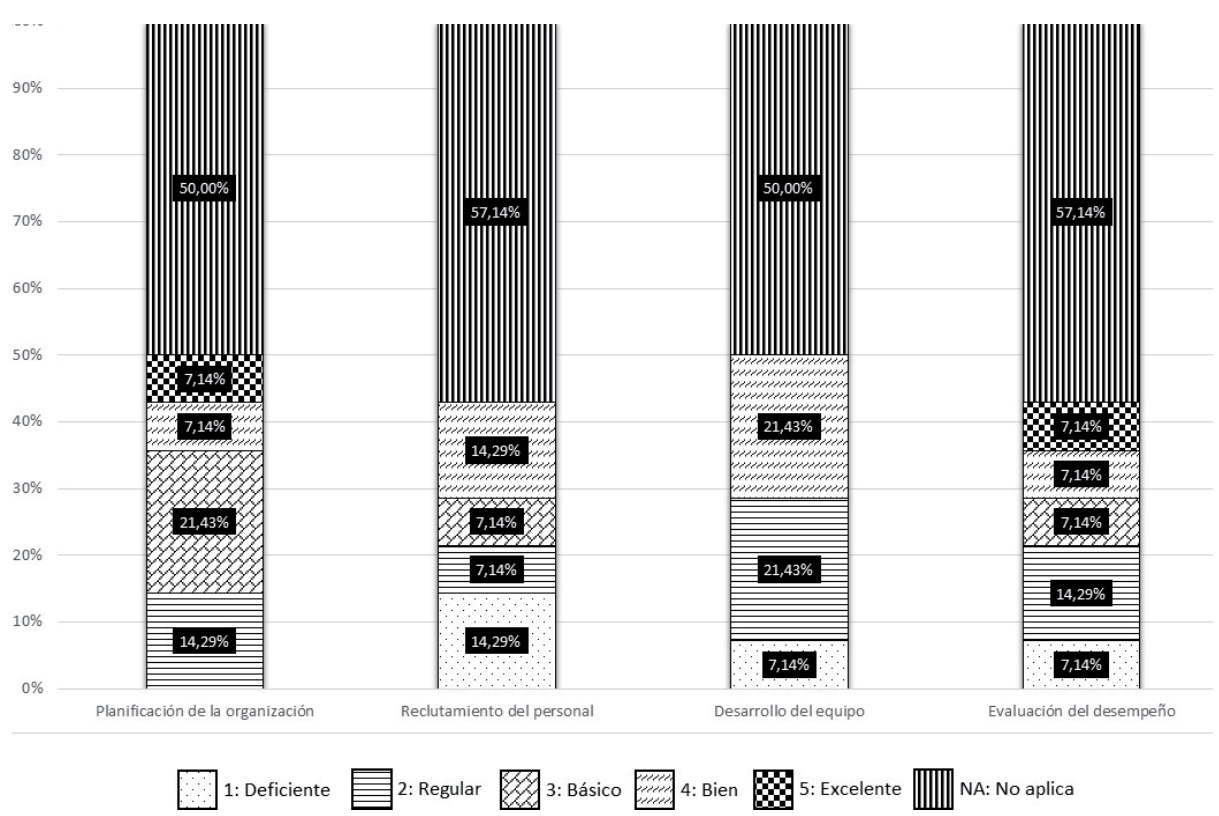

organización empresarial y comercio exterior), por lo que se esperaba un mejor desempeño en este aspecto, al ser muchos de ellos expertos en motivación, supervisión de equipos, técnicas de negociación y técnicas de optimización.

Al no realizarse de forma general una evaluación del desempeño de los participantes de los equipos de proyectos, quedan en evidencia fallas relacionadas con la gestión del desarrollo del equipo antes mencionada, ya que no es posible motivar o impulsar el trabajo sin el feedback requerido por parte del gerente del proyecto.

En el caso de las comunicaciones (presentada en la figura 3), casi el $40 \%$ de los consultados consideran que realizaron una gestión buena planificando las comunicaciones de los proyectos realizados (superando esa cifra si se incluyen a aquellos que consideran haber realizado una excelente gestión) al identificar a los involucrados y sus necesidades de información. Todo esto a pesar de los aparentes problemas de comunicación con parte de los involucrados (específicamente con el equipo de proyectos, dado los resultados obtenidos en los criterios vinculados con los recursos humanos).

La mayoría de los profesores consideraron que para sus casos particulares no era necesario distribuir la información (posiblemente por la individualización exigida por la universidad para profesores noveles o fuera del escalafón universitario que ya fue comentada), por lo que ellos eran quienes realizaban todas las actividades del proyecto, no implicando que debieran comunicarse a gran escala durante la ejecución de estos. Sin embargo, la mitad de los profesores reportaron una gestión entre deficiente, regular y básica para los reportes de progreso, sirvieran estos reportes como insumo propio o para compartirlos con un equipo de trabajo, perjudicando sin duda la ejecución y el proceso de monitoreo y control del avance del proyecto.

También la mitad de los consultados indicaron una gestión mayoritariamente agrupada entre las categorías deficiente, regular y básico a la hora de realizar el cierre administrativo de las comunicaciones, por lo que no se recogieron las lecciones aprendidas fundamentales para la formulación y desarrollo de futuros proyectos. 
Figura 3. Gestión de las comunicaciones en proyectos realizados por profesores del Departamento de Tecnología de Servicios - USB

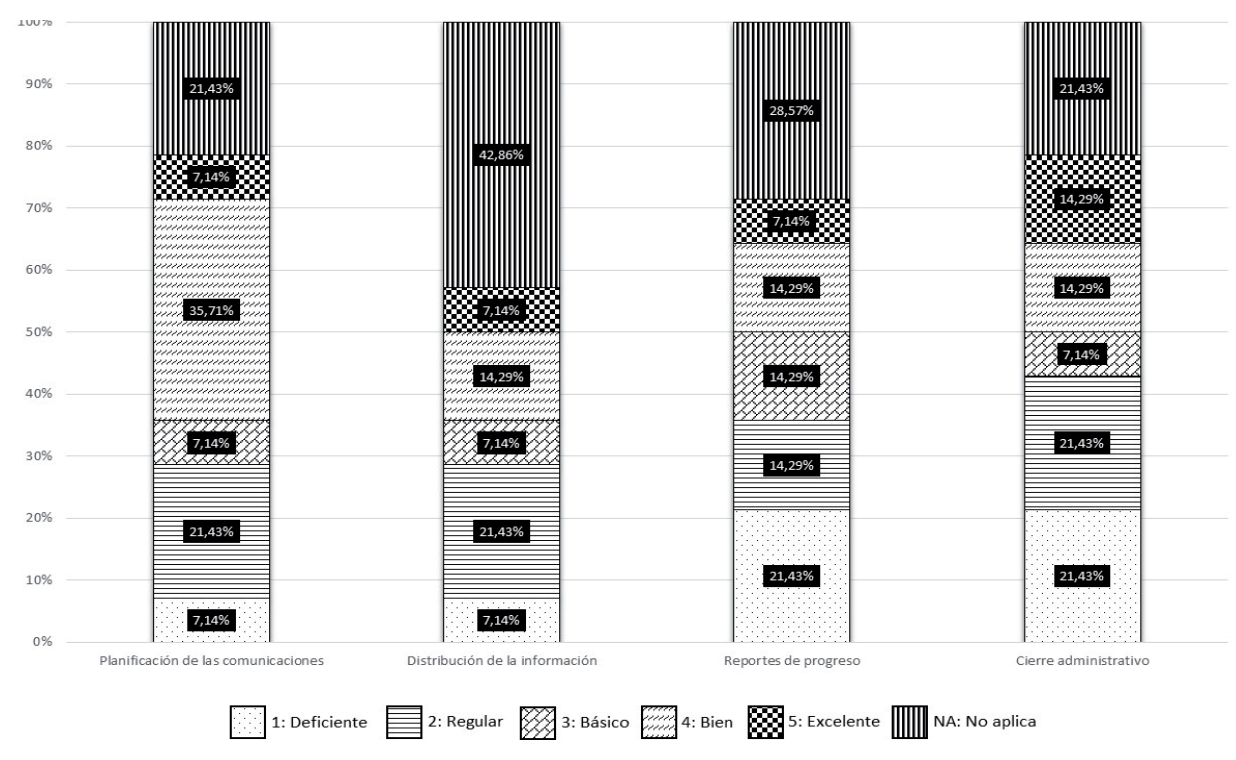

Si se analiza la gestión de los riesgos (figura 4), es el área de conocimiento con el peor desempeño autoevaluado por los profesores, siendo casi dos tercios de estos los que indicaron que en su gestión se identificaron riesgos. El calificarlos y diseñar un plan de respuestas fue entre deficiente y regular, por lo que de entrada los proyectos se encontraron vulnerables a estos, al no identificarse factores que pudiesen afectar las actividades planificadas, así como los resultados de los mismos. No se evaluó tampoco la probabilidad de ocurrencia e impacto de riesgos en los proyectos - principalmente porque no se identificaron- $y$, por lo tanto, no hay un plan de respuesta adecuado.

Más de la mitad de los profesores también autoevaluaron como deficiente y regular la gestión del control de respuesta ante riesgos, por lo que no hicieron seguimiento a los que habían identificado, ni actuado frente a ellos.

Figura 4. Gestión de los riesgos en proyectos realizados por profesores del Departamento de Tecnología de Servicios - USB

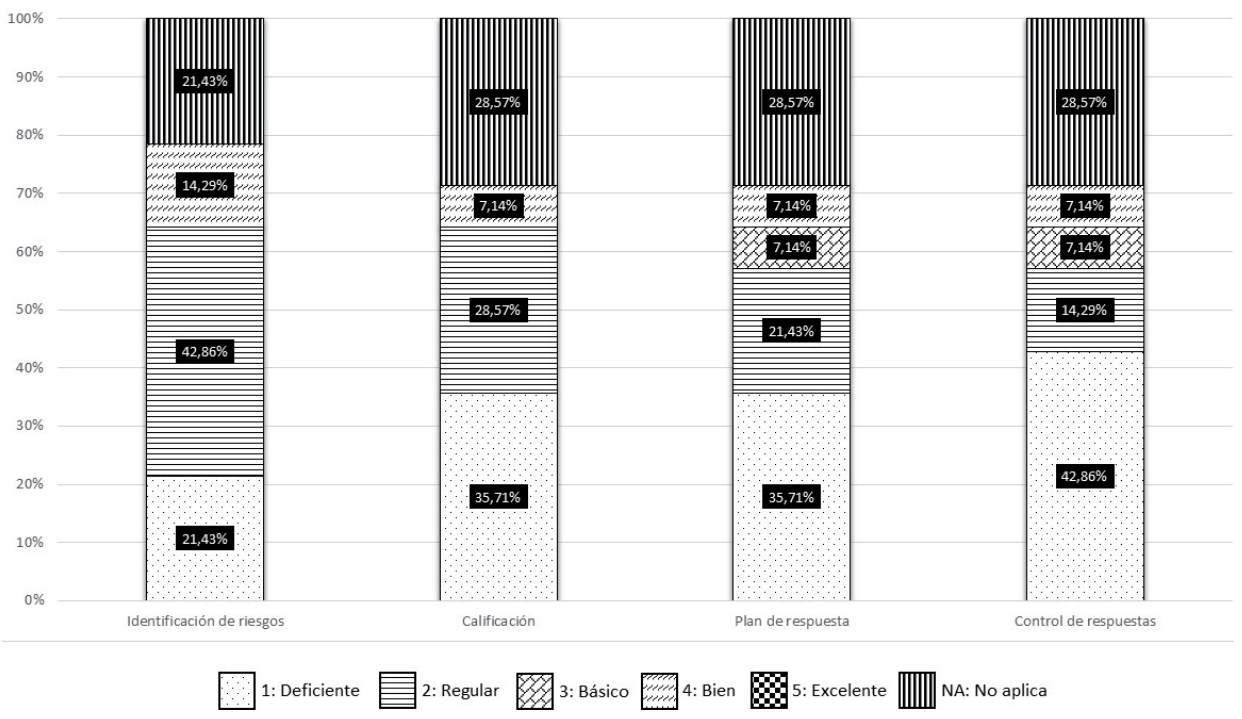


Por su parte, en la gestión de las adquisiciones, cuyos resultados pueden observarse en la figura 5, hay que destacar que la mitad de los profesores se autoevaluaron con una gestión entre básica y bien a la hora de diseñar un plan de compras que les permitiera identificar todos los recursos requeridos por sus proyectos. Este buen resultado se debe en parte a la exigencia de la universidad para aprobar recursos financieros a los proyectos sujetos a un plan de compra justificado pero limitado para convocatorias especiales sujetas a disponibilidad presupuestaria, junto con la escasez existente en el país, lo que hizo que no siempre se adquiriera o contratara todo lo plasmado en el plan.

De igual modo, como básico y bien, se evaluaron más de la mitad de los profesores en la gestión realizada en el plan de requerimientos y el ciclo de solicitación, lo que implica que la mayoría de los proyectos realizados ajustaron las compras y contrataciones a las necesidades detectadas tras identificar las mejores opciones de adquisición.

Casi dos tercios de los profesores señalan que tuvieron una administración de contratos calificada como deficiente, regular y básica, ya que no se hizo el seguimiento necesario a los mismos. También dos tercios de la muestra afirmaron no hacer un cierre adecuado a los contratos ni la rendición de cuentas relacionada, a pesar de ser exigencia de la universidad para introducir nuevos proyectos y solicitar nuevo financiamiento.

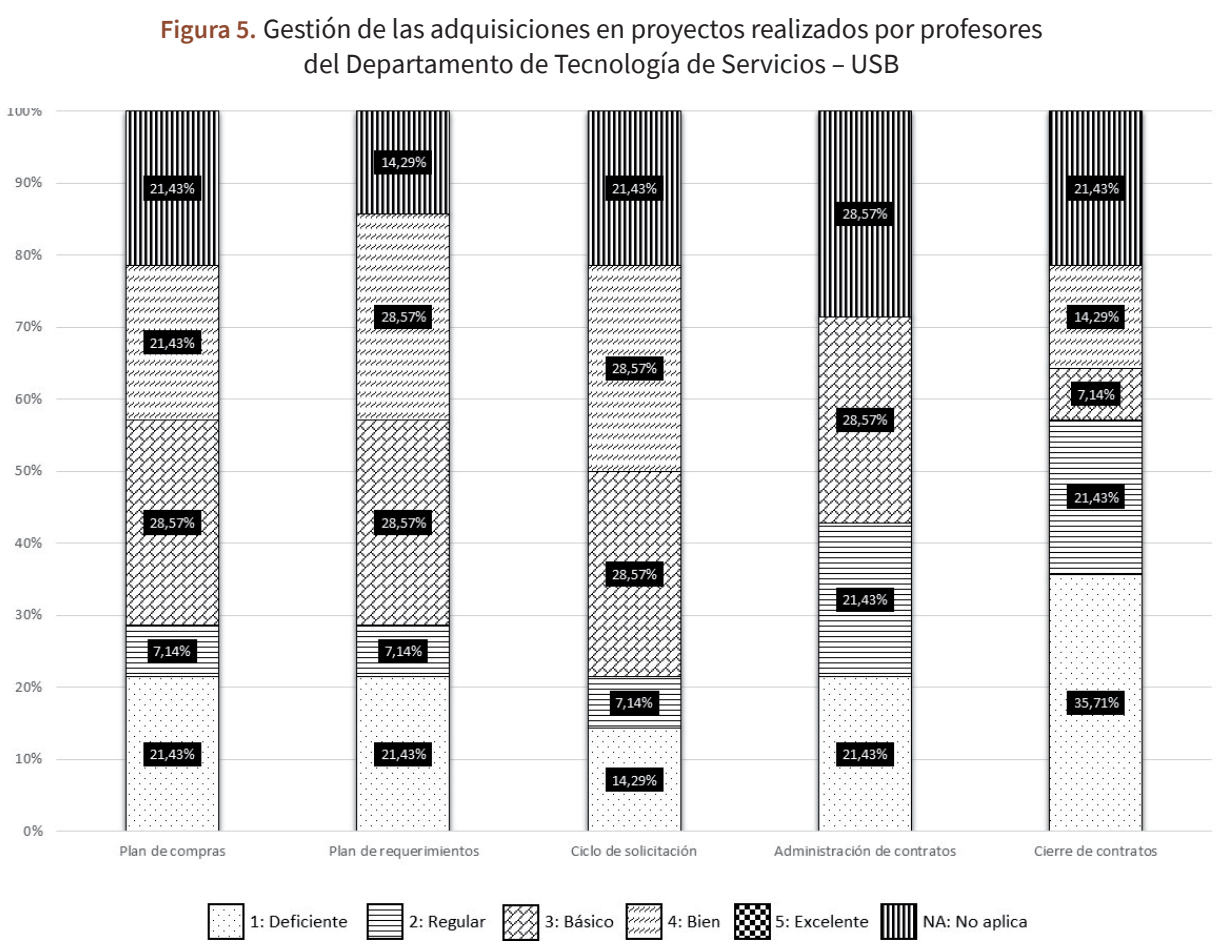

En el caso de la gestión de los interesados, cuyos resultados se aprecian en la figura 6, más de la mitad de los consultados consideran que definieron el alcance del proyecto de manera apropiada, estimando todos los aspectos que deben incluirse en el mismo y, por lo tanto, incorporando la opinión de la mayor cantidad de involucrados. No obstante, hay que destacar que más del 40\% respondió que, por el contrario, su definición del alcance fue deficiente o regular, lo que obviamente les debió traer problemas a la hora de ejecutar los proyectos, tanto con sus entregables como con algunos de los interesados. 
Alrededor de dos tercios de los profesores gestionan el tiempo de algún modo, haciéndolo la mayoría de forma básica. Dentro de ese porcentaje, hay quienes afirman hacerlo bien e, incluso, de forma excelente a la hora de asignar tiempos de ejecución conjunta con los interesados. Por otro lado, casi el $80 \%$ de los profesores utilizó un cronograma para programar sus actividades, haciendo la mayoría un uso básico y bueno dentro de ese porcentaje, incorporando a los interesados para darle coherencia a lo planificado.

En el caso de los costos, la mitad de los profesores los estimaron de forma apropiada, usando al menos de forma básica o buena los métodos adecuados y considerando toda la información disponible que les fue suministrada por los involucrados en los proyectos.

Los indicadores de gestión no fueron utilizados por casi un tercio de los consultados a la hora de planificar la calidad del resultado final, aunque un porcentaje igual afirmó utilizarlo y hacerlo bien. Hay que destacar que las especificaciones de calidad de los resultados deben satisfacer a los involucrados, por lo que esta gestión es de suma importancia en los proyectos.

Los profesores, en su papel de gerente integrador de procesos y áreas de conocimiento de los proyectos que llevaron a cabo, descuidaron desde ese punto de vista a los interesados, ya que la mitad respondió que, en este caso, su gestión puede calificarse como de regular a deficiente respecto a los involucrarlos.

Figura 6. Gestión de los interesados en proyectos realizados por profesores del Departamento de Tecnología de Servicios - USB

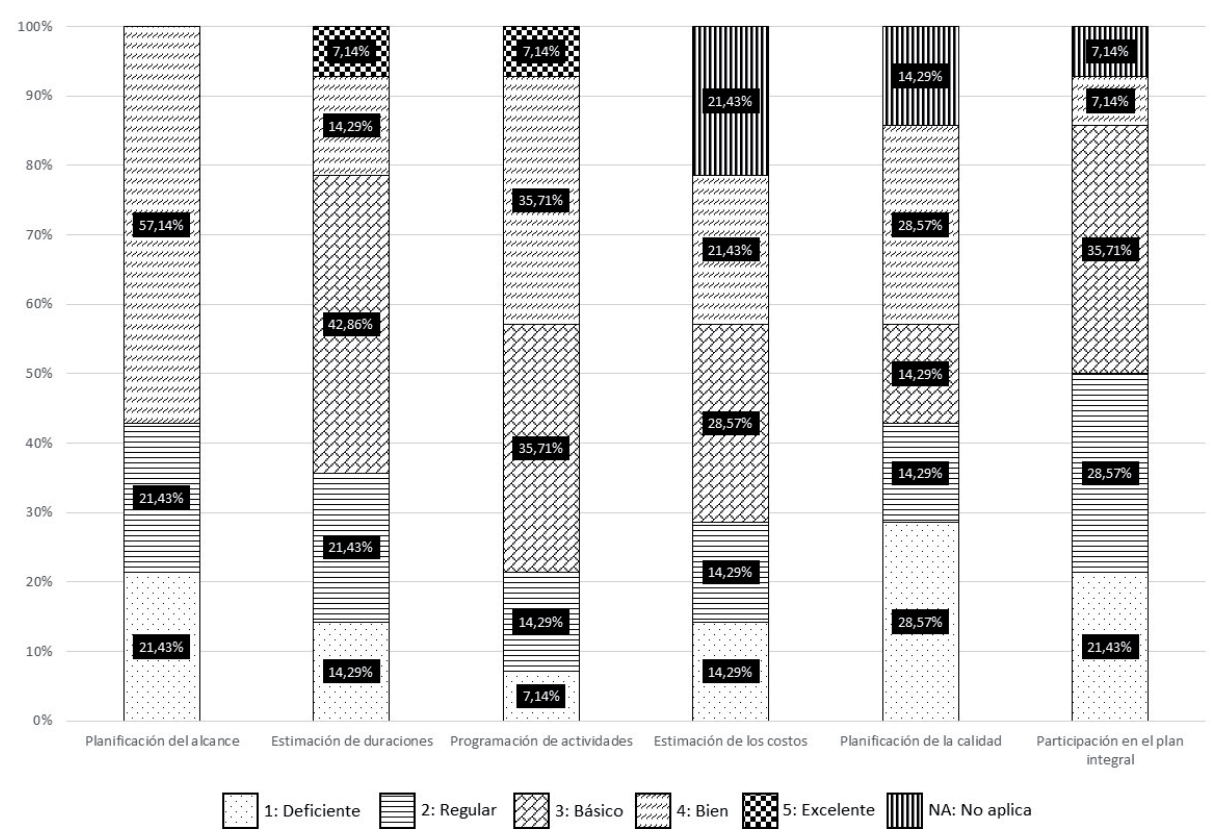

\section{CONCLUSIONES}

El diagnóstico realizado a los profesores del Departamento de Tecnología de Servicios de la Universidad Simón Bolívar de Venezuela reveló que en cuanto a la gestión de las áreas de conocimiento de apoyo a la gerencia de proyectos, presentan deficiencias a la hora de aplicar metodologías, técnicas y herramientas, al menos las más adecuadas, para los proyectos que realizan. Esto puede ser por desconocimiento u omisión, dejando margen en muchos casos para la aplicación de estas de forma intuitiva. Una adecuada gestión es 
fundamental para los proyectos realizados en las universidades, ya que están vinculados a investigaciones particulares de profesores y alumnos y su efecto multiplicador, de todo aquello que se realiza dentro de estas instituciones educativas hacia la sociedad, es significativo, por lo que gestionar los proyectos considerando todos sus procesos y áreas de conocimiento es algo que debe ser prioritario.

Si se considera la gestión de los recursos humanos realizada por los profesores del departamento estudiado en sus proyectos, hay que destacar que aunque la mayoría de ellos no contaron con equipos de trabajo, quienes sí lo hicieron manifestaron fallas al no definir e integrar roles y responsabilidades, así como también a la hora desarrollar sus capacidades y evaluar el desempeño de estos, lo que sin duda dificulta organizar y dirigir un equipo de trabajo y, mucho menos, que este personal contribuya y participe con las expectativas claras desde el inicio, durante y hasta el final de sus labores.

En cuanto a la gestión de las comunicaciones, a pesar de haberse planificado la misma considerando a todos los involucrados y las necesidades de información detectadas, las deficiencias se presentaron tanto a la hora de distribuir la información y reportar el progreso, como haciendo el cierre administrativo, lo que implica que debieron existir problemas a la hora de ejecutar y hacer el monitoreo y control de los proyectos, así como a la hora de recoger las lecciones aprendidas fundamentales para el éxito de futuros proyectos a realizar.

La gestión de riesgos fue la peor autoevaluada por los profesores, ya que las deficiencias abarcan todos los procesos de gestión involucrados, ya que no se identificaron ni calificaron los riesgos y tampoco se diseñó un plan de respuestas adecuado, afectando tanto a las actividades planificadas como a los resultados esperados.

Por su parte, la gestión de las adquisiciones permitió, aunque sea de forma básica, identificar los recursos necesarios, así como gestionar el plan de requerimientos y el ciclo de solicitación ajustado a las necesidades identificadas y a las opciones de compras o de contratación detectadas. Sin embargo, la administración y cierre de los contratos fue deficiente, tanto para la rendición de cuentas como para dejar claras las expectativas con quienes se involucraron en dichos contratos.

Finalmente, en el caso de la gestión de los interesados, la cual fue evaluada en las gestiones antes reseñadas (considerando adicionalmente criterios vinculados con las áreas medulares e integradora), la opinión de los interesados se consideró clave a la hora de definir el alcance, así como para asignar tiempos a las actividades, darle coherencia al cronograma diseñado y, también, a la hora de estimar los costos. No obstante, no se utilizaron indicadores de gestión que contemplaran la opinión de los interesados y la autoevaluación revela que, de forma general, hubo descuido en las actividades integradoras a la hora de considerarlos.

La información obtenida de este trabajo no es útil solamente para el departamento estudiado y el resto de la universidad, si no que puede servir de referencia para que en otras universidades e institutos comprendan la importancia de gestionar las áreas de conocimiento de la gerencia de proyectos. Retomando el caso puntual que se analiza en este artículo, es importante aclarar que la actual situación del país y su incidencia en la universidad, implican un gran reto para llevar a cabo las actividades docentes, de investigación y de extensión (pilares fundamentales universitarios), por lo que los proyectos realizados, a pesar de la vocación manifestada por el cuerpo docente, no escapan a la escasez, a la hiperinflación y a las deficiencias de servicios e infraestructuras. Sin embargo, dado el impacto social de los proyectos realizados desde la universidad, el diagnóstico realizado permitirá tomar acciones correctivas a través de los planes de desarrollo profesional de la institución, permitiendo plantear la realización de talleres de formación en el área con los cuales subsanar las deficiencias diagnosticadas. 


\section{REFERENCIAS}

Arias, F. (2012). El proyecto de investigación: introducción a la investigación científica. Editorial Episteme.

Baca, G. (2013). Evaluación de proyectos. McGraw-Hill.

Estrada, J. (2015). Análisis de la gestión de proyectos a nivel mundial. Palermo Business Review, (12), 61-98.

Fàbregues, S., Meneses, J., Rodríguez-Gómez, D., y Paré, M. (2016) Técnicas de investigación social y educativa. Editorial UOC.

Gómez, M. (2011). Aproximación a la visión del PMI acerca de las competencias requeridas por los gerentes de proyecto como componente fundamental de la dimensión humana del capital intelectual. Revista CONHISREMI, 7(1), 21-31.

Holloway, J., Bryde, D., y Joby, R. (2015). A practical guide to dealing with difficult stakeholders. Gower Publishing.

Marchewka, J. (2015). Information technology project management. Providing measurable organizational value. John Wiley \& Sons.

Ollé, C., y Cerezuela, B. (2017). Gestión de proyectos paso a paso. Editorial UOC.

Palacios, L. (2009). Principios esenciales para realizar proyectos. Un enfoque latino. Universidad Católica Andrés Bello.

Prieto, J. (2015). Proyectos: enfoque gerencial. Ecoe ediciones.

Project Management Institute (2013). Guía de los fundamentos para la dirección de proyectos (Guía del PMBOK). Project Management Institute.

Romano, G., y Yacuzzi, E. (2011). Elementos de la gestión de proyectos. Serie documentos de trabajo de la Universidad del CEMA, 449, 1-32.

Santos, M., Lorenzo, M., y Priegue, D. (2013). Red conectando a los profesores para el desarrollo de la interculturalidad. Revista Educación XX1, 16(1), 63-84.

Sebastián, M., Arenas, J. y Claver, J. (2017). Oficina técnica y gestión de proyectos. Universidad Nacional de Educación a Distancia.

Turner, R. (2016). Handbook of project management. Routledge.

Ugas, L. (2008). La gestión de los proyectos en las empresas del sector energético. Caso: ENELVEN - CARBOZULIA. Télématique Revista Electrónica de Estudios Telemáticos, 7(2), 70-97.

Wallace, W. (2014). Gestión de proyectos. Edinburgh Business School.

Wilson, R. (2015). Mastering project management strategy and processes. Proven methods to meet organizational goals. Pearson Education. 